\title{
THE GENES REgUlating MAINTENANCE OF CELlUlaR PROTEIN LOCATION ARE DIFFERENTIALLY EXPRESSED IN PORCINE EPITHELIAL OVIDUCTAL CELLS DURING LONG- TERM IN VITRO CULTIVATION
}

Katarzyna Stefańska ${ }^{1}$, Ievgenia Kocherova ${ }^{2}$, Sandra Knap ${ }^{1}$, Magdalena Kulus ${ }^{3}$, Piotr Celichowski ${ }^{1}$, Michal Jeseta ${ }^{4}$

\begin{abstract}
The oviduct is a part of female reproductive tract that is essential for successful fertilization and early embryo development. It is lined with epithelium consisting of two types of cells: ciliated and secretory. The primary function of ciliated oviductal epithelial cells (OECs) is to support the transport of gametes and embryos through the ovary, whereas secretory OECs produce components of the oviductal fluid. Undoubtedly, the oviductal epithelium plays a major part in the early aspects of pregnancy development, from providing an optimal environment for gametes and embryos to supporting fertilization. Therefore, our aim was to gain a better insight into the genetic changes underlying function of these cells. We have harvested OECs from crossbred gilts $(n=45)$, at the age of about nine months and which displayed two regular estrous cycles, and established long-term primary culture of porcine OECs. Microarray analysis was utilized to determine differentially expressed genes during day 1, 7, 15 and 30 of cultivation, with our results revealing54 differentially expressed genes belonging to three ontology groups: „maintenance of location”, „maintenance of protein location" and "maintenance of protein location in cell”. Since the biochemistry and morphology of epithelial cells may change during long term cultivation, we conclude that our results are a reflection of these changes and help to shed a light on porcine OECs properties in in vitro environment.
\end{abstract}

Running title: Maintenance of cellular protein location in porcine epithelial oviductal cells

Keywords: oviductal epithelial cells, culture, protein, location

\footnotetext{
${ }^{1}$ Department of Histology and Embryology, Poznan University of Medical Sciences, Poznan, Poland

${ }^{2}$ Department of Anatomy, Poznan University of Medical Sciences, Poznan, Poland

${ }^{3}$ Veterinary Centre, Nicolaus Copernicus University in Torun, Torun, Poland

${ }^{4}$ Department of Obstetrics and Gynaecology, University Hospital and Masaryk University, Brno, Czech Republic

* Correspondence: jeseta@gmail.com

Full list of author information is available at the end of article
} 


\section{Introduction}

The female reproductive tract is composed of the oviduct, uterus, cervix and vagina. The oviduct derives from the Müllerian duct and plays vital role in successful monospermic fertilization and female fertility. It has two muscle layers and a mucous lining and is covered with two types of epithelial cells: ciliated and non-ciliated secretory. The oviduct consists of four regions: the infundibulum, the ampulla, which is the site of fertilization, the isthmus and the uterotubal junction. It is involved in sperm guidance to fertilization site via the mechanisms of rheotaxis, thermotaxis and chemotaxis. Moreover, the oviductal epithelial cells (OECs) physically interact with cumulus-oocyte complexes (COCs), as well as with the sperm, which results in sperm hyperactivation through the CatSper activation on the flagella [1].

Both ciliated and secretory oviductal epithelial cells play an invaluable role in the female fertility. The proportion of secretory OECs increases towards the isthmus, while the ciliated cells decrease. Secretory OECs produce components of the oviductal fluid, while the primary function of ciliated OECs is to support the transport of gametes and embryos through the ovary. They generate the movement of the oviductal fluid, with and the direction of the flow depending on the species; in pigs it is towards the ovary [1]. Oviductal fluid contains glucose, lactate, pyruvate, amino acids, embryotropic and growth factors and other molecules essential for both gamete and embryo survival. However, the composition of oviductal fluid and secretory activity of the oviductal epithelial cells changes during the estrous cycle in response to hormone levels [2].

Pre-implantation embryo development is affected by oviductal epithelial cells as well. Studies conducted by White et al. clearly show that the coculture of primary porcine OECs with early embryos promotes blastocyst cleavage [3]. Similar results were obtained in sheep, mouse, cattle and human, which suggests that OECs provide an optimal environment for early embryo development [1].

Since the oviduct and OECs undoubtedly play major part in early aspects of pregnancy development, from providing an optimal environment for gametes and embryos to supporting fertilization, our aim was to gain a better insight into genetic changes that underlie these cells' function. We established a long-term primary culture of porcine OECs and performed microarray analysis, checking gene expression in specific time periods. Our results revealed a set of genes belonging to "maintenance of location", "maintenance of protein location in cell" and "maintenance of protein location" that were differentially expressed, indicating that they may be possible marker genes of biochemical and morphological changes in OECs.

\section{Material and Methods \\ Animals}

In this study, crossbred gilts $(n=45)$ at the age of about nine months and which displayed at least two regular estrous cycles were collected from a commercial herd. All the animals were checked daily for estrus behavior and were slaughtered after reaching the anestrus phase of the estrus cycle. The uteri were then transported to the laboratory within 30 $\min$ at $38^{\circ} \mathrm{C}$.

\section{Oviductal epithelial cells (OECs) selection and culture}

Oviducts were washed twice in Dulbecco's phosphate buffered saline (PBS) $(137 \mathrm{mM} \mathrm{NaCl}, 27 \mathrm{mM}$ $\mathrm{KCl}, 10 \mathrm{mM} \mathrm{Na} 2 \mathrm{HPO} 4,2 \mathrm{mM} \mathrm{KH} 2 \mathrm{PO} 4, \mathrm{pH}$ 7.4). Epithelial cells were removed using sterile surgical blades. Then, the epithelium was incubated with collagenase I (Sigma Aldrich, Madison, USA), 1mg/ $\mathrm{mL}$ in Dubecco's modified Eagle's medium (DMEM; Sigma Aldrich, Madison, USA) for $1 \mathrm{~h}$ at $37 \mathrm{oC}$. The cell suspension obtained from this digestion was filtered through $40 \mu \mathrm{m}$ pore size strainer to remove blood and single cells. The residue was collected by rinsing the strainer with DMEM. The cells were then centrifuged ( $200 \mathrm{x} \mathrm{g}, 10 \mathrm{~min}$.). Next, they were washed in PBS and centrifuged again. Later, they were incubated with $0.5 \%$ Trypsin/EDTA (Sigma Aldrich, Madison, USA) at $37 \mathrm{oC}$ for $10 \mathrm{~min}$. The reaction was stopped with fetal calf serum (FCS; Sigma Aldrich, Madison, USA). After incubation, the cells were filtered and centrifuged for the last time. The final cell pellet was suspended in DMEM, supplemented with $10 \% \mathrm{FCS}, 100 \mathrm{U} / \mathrm{mL}$ penicillin, $100 \mu \mathrm{g} / \mathrm{mL}$ streptomycin and $1 \mu \mathrm{g} / \mathrm{mL}$ amphotericin B. The cells were cultured at $37^{\circ} \mathrm{C}$ in a humidified atmosphere of $5 \% \mathrm{CO} 2$. Once the OEC cultures attained $70-80 \%$ confluency, they were passaged by washing with PBS, digested with $0.025 \%$ Trypsin/ EDTA, neutralized by a $0.0125 \%$ trypsin inhibitor (Cascade Biologics, Portland, USA), centrifuged, and resuspended at a seeding density of $2 * 10^{4}$ cells/ $\mathrm{cm} 2$. The culture medium was changed every three days. The culture was maintained for 30 days.

\section{RNA extraction from oviductal epithelial cells (OECs)}

Oviductal epithelial cell were pooled and harvested $24 \mathrm{~h}, 7$ days, 15 days and 30 days after the beginning of culture. Total RNA was extracted from the samples using TRI Reagent (Sigma, St Louis, MO, USA) and RNeasy MinElute cleanup Kit (Qiagen, Hilden, Germany). The total mRNA amount was determined from the optical density at $260 \mathrm{~nm}$, and the RNA purity was estimated using the $260 / 280$ $\mathrm{nm}$ absorption ratio (higher than 1.8) (NanoDrop spectrophotometer, Thermo Scientific, ALAB, Po- 
land). The RNA integrity and quality were checked on a Bioanalyzer 2100 (Agilent Technologies, Inc., Santa Clara, CA, USA). The resulting RNA integrity numbers (RINs) were between 8.5 and 10 with an average of 9.2 (Agilent Technologies, Inc., Santa Clara, CA, USA). The RNA in each sample was diluted to a concentration of $100 \mathrm{ng} / \mu \mathrm{l}$ with an OD260/OD280 ratio of 1.8/2.0. From each RNA sample, 100 ng of RNA was taken for microarray expression assays.

\section{Microarray expression analysis and statistics}

Total RNA (100 ng) from each pooled sample was subjected to two rounds of sense cDNA amplification (Ambion ${ }^{\circledR}$ WT Expression Kit). The obtained cDNA was used for biotin labeling and fragmentation using Affymetrix GeneChip $®$ WT Terminal Labeling and Hybridization (Affymetrix, Santa Clara, CA, USA). Biotin-labeled fragments of cDNA $(5.5 \mu \mathrm{g})$ were hybridized to the Affymetrix $\AA$ Porcine Gene 1.1 ST Array Strip $\left(48^{\circ} \mathrm{C} / 20 \mathrm{~h}\right)$. Microarrays were then washed and stained, according to the technical protocol, using the Affymetrix GeneAtlas Fluidics Station. The array strips were scanned employing the Imaging Station of the GeneAtlas System. Preliminary analysis of the scanned chips was performed using Affymetrix GeneAtlas ${ }^{\mathrm{TM}}$ Operating Software. The quality of gene expression data was confirmed according to the quality control criteria provided by the software. The obtained CEL files were imported into downstream data analysis software.

All of the presented analyses and graphs were compiled using Bioconductor and R programming languages. Each CEL file was merged with a description file. To correct background, normalize, and summarize results, we used the Robust Multiarray Averaging (RMA) algorithm. To determine the statistical significance of the analyzed genes, moderated t-statistics from the empirical Bayes method were performed. The obtained p-value was corrected for multiple comparisons using Benjamini and Hochberg's false discovery rate. Selection of significantly altered genes was based on a p-value beneath 0.05 and expression higher than two-fold.

Differentially expressed genes were subjected selection by examination of genes involved in regulation of maintenance of cellular protein location. The differentially expressed gene list (separated for up- and down-regulated genes) was uploaded to the DAVID software (Database for Annotation, Visualization and Integrated Discovery) [4], where genes belonging to the terms of all three Gene Ontologies (GOs) of interest were extracted. Expression data of these genes was also subjected to a hierarchical clusterization procedure, with their expression values presented as a heat map.

Subsequently, we analyzed the relation between the genes belonging to the chosen GO terms using the GOplot package [5]. The GoPlot package had calculated the z-score: the number of up- regulated genes minus the number of down- regulated genes divided by the square root of the count. This information allowed to estimate the change course of each gene-ontology term.

Interactions between differentially expressed genes/proteins belonging to the studied gene ontology groups were investigated by the STRING10 software (Search Tool for the Retrieval of Interacting Genes) [6]. The list of gene names was used as a query for interaction prediction. The search criteria were based on co-occurrences of genes/proteins in scientific texts (text mining), co-expression, and experimentally observed interactions. The results of such analyses generated a gene/protein interaction network where the intensity of the edges reflected the strength of the interaction score.

Finally, the functional interactions between genes that belongs to the chosen GO BP terms were investigated by the REACTOME FIViz application to the Cytoscape 3.6.0 software. The ReactomeFIViz app is designed to find pathways and network patterns related to cancer and other types of diseases. This app accesses the pathways stored in the Reactome database, allowing to perform pathway enrichment analysis for a set of genes, visualize hit pathways using manually laid-out pathway diagrams directly in Cytoscape, and investigate functional relationships among genes in hit pathways. The app can also access the Reactome Functional Interaction (FI) network, a highly reliable, manually curated pathway-based protein functional interaction network covering over $60 \%$ of human proteins.

\section{Ethical approval}

The research related to animal use has been complied with all the relevant national regulations and instructional policies for the care and use of animals. Bioethical Committee approval no. 32/2012.

\section{Results}

Whole transcriptome profiling with Affymetrix microarrays allows us to analyze the gene expression changes between 7,15 and 30 days of porcine oviductal epithelial cell culture. Using Affymetrix ${ }^{\circledR}$ Porcine Gene 1.1 ST Array Strip, we have examined the expression of 12257 transcripts. Genes with fold change higher than abs (2) and with corrected p-value lower than 0.05 were considered as differentially expressed. This set of genes consists of 2533 different transcripts.

DAVID (Database for Annotation, Visualization and Integrated Discovery) software was used for extraction of gene ontology biological process terms (GO BP) that contain differently expressed transcripts. Up and down regulated gene sets were subjected to the DAVID search separately and only gene sets with adj. $p$ value lower than 0.05 were selected. The DAVID software analysis showed that the differently expressed genes belonged to 657 Gene ontology terms. 

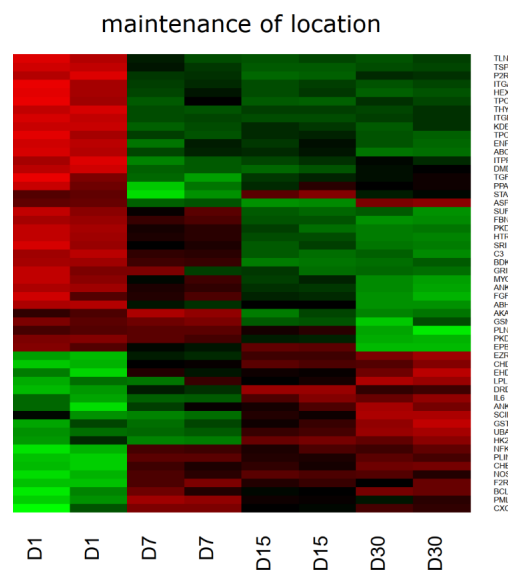

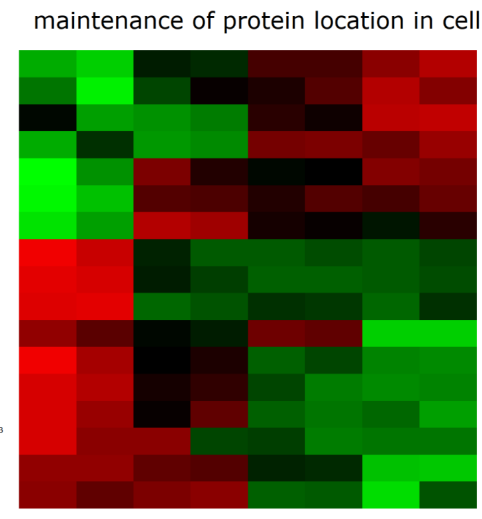

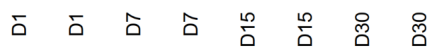

maintenance of protein location

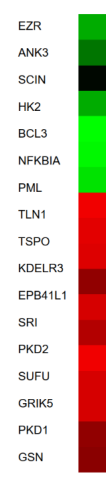

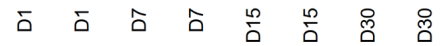

FIGURE 1 Heat map representation of differentially expressed genes belonging to the to "maintenance of location", maintenance of protein location in cell" and " maintenance of protein location" GO BP terms. Arbitrary signal intensity acquired from microarray analysis is represented by colours (green, higher; red, lower expression). Log2 signal intensity values for any single gene were resized to Row Z-Score scale (from -2 , the lowest expression to +2 , the highest expression for single gene). The 20 genes selected to this publication was marked green for upregulation and red for downregulation

TABLE 1 Gene symbols, fold change in expression ratio, Entrez gene IDs, corrected p values and mean value of fold change ratio of studied genes

\begin{tabular}{|c|c|c|c|c|c|c|c|c|}
\hline $\begin{array}{c}\text { GENE } \\
\text { SYMBOL }\end{array}$ & $\begin{array}{l}\text { RATIO } \\
\text { D7/D1 }\end{array}$ & $\begin{array}{c}\text { RATIO } \\
\text { D15/D1 }\end{array}$ & $\begin{array}{c}\text { RATIO } \\
\text { D30/D1 }\end{array}$ & $\begin{array}{c}\text { ADJUSTED } \\
\text { P.VALUE } \\
\text { D7/D1 }\end{array}$ & $\begin{array}{c}\text { ADJUSTED } \\
\text { P.VALUE } \\
\text { D15/D1 }\end{array}$ & $\begin{array}{c}\text { ADJUSTED } \\
\text { P.VALUE } \\
\text { D30/D1 }\end{array}$ & $\begin{array}{l}\text { ENTREZ } \\
\text { GENE ID }\end{array}$ & $\begin{array}{l}\text { MEAN } \\
\text { RATIO }\end{array}$ \\
\hline PLIN2 & $-4,907$ & $-3,554$ & $-4,593$ & $1,11^{*} 10^{\wedge} 5$ & $1,34 * 10^{\wedge} 5$ & $3,34 * 10^{\wedge} 6$ & 397402 & $-4,352$ \\
\hline UBASHЗB & $-1,211$ & $-3,527$ & $-6,541$ & $2,19 * 10^{\wedge} 1$ & $5,65^{*} 10^{\wedge} 5$ & $4,84^{*} 10^{\wedge} 6$ & 100521108 & $-3,760$ \\
\hline CHD7 & $-2,636$ & $-3,935$ & $-4,447$ & $2,97 * 10^{\wedge} 4$ & $3,61^{*} 10^{\wedge} 5$ & $1,51^{*} 10^{\wedge} 5$ & 100157083 & $-3,673$ \\
\hline DRD2 & $-1,904$ & $-4,799$ & $-2,946$ & $1,12^{*} 10^{\wedge} 3$ & $1,05^{*} 10^{\wedge} 5$ & $4,14^{*} 10^{\wedge} 5$ & 100624857 & $-3,216$ \\
\hline CXCL10 & $-4,137$ & $-2,237$ & $-3,015$ & $2,16^{*} 10^{\wedge} 3$ & $2,59 * 10^{\wedge} 2$ & $4,92 * 10^{\wedge} 3$ & 494019 & $-3,130$ \\
\hline NoS1 & $-2,717$ & $-2,984$ & $-2,679$ & $2,24 * 10^{\wedge} 4$ & $9,95 * 10^{\wedge} 5$ & $1,15^{*} 10^{\wedge} 4$ & 100520934 & $-2,794$ \\
\hline EZR & $-1,728$ & $-2,489$ & $-3,404$ & $1,74 * 10^{\wedge} 3$ & $1,06^{*} 10^{\wedge} 4$ & $1,49 * 10^{\wedge} 5$ & 100152815 & $-2,540$ \\
\hline NFKBIA & $-2,378$ & $-2,256$ & $-2,464$ & $3,20 * 10^{\wedge} 4$ & $3,17^{*} 10^{\wedge} 4$ & $1,30 * 10^{\wedge} 4$ & 406188 & $-2,366$ \\
\hline ANK3 & $-1,644$ & $-2,184$ & $-3,063$ & $4,87^{*} 10^{\wedge} 2$ & $6,10^{*} 10^{\wedge} 3$ & $7,47^{*} 10^{\wedge} 4$ & 100154687 & $-2,297$ \\
\hline$F 2 R$ & $-2,545$ & $-2,149$ & $-2,185$ & $6,98 * 10^{\wedge} 4$ & $1,46^{*} 10^{\wedge} 3$ & $9,88^{*} 10^{\wedge} 4$ & 100521609 & $-2,293$ \\
\hline ITGAV & 5,815 & 6,507 & 6,738 & $1,26^{*} 10^{\wedge} 4$ & $6,01 * 10^{\wedge} 5$ & $3,42 * 10^{\wedge} 5$ & 397285 & 6,353 \\
\hline C3 & 2,665 & 8,332 & 9,436 & $6,56^{*} 10^{\wedge} 4$ & $1,06^{*} 10^{\wedge} 5$ & $4,89 * 10^{\wedge} 6$ & 397072 & 6,811 \\
\hline$P 2 R X 7$ & 6,165 & 8,522 & 5,795 & $2,69 * 10^{\wedge} 5$ & $6,72^{*} 10^{\wedge} 6$ & $1,03 * 10^{\wedge} 5$ & 497623 & 6,828 \\
\hline$A B C A 1$ & 6,313 & 5,557 & 10,336 & $3,95 * 10^{\wedge} 5$ & $3,12^{*} 10^{\wedge} 5$ & $4,36^{*} 10^{\wedge} 6$ & 100152112 & 7,402 \\
\hline ANK2 & 3,085 & 6,719 & 15,991 & $1,37^{*} 10^{\wedge} 4$ & $6,90^{*} 10^{\wedge} 6$ & $7,60^{*} 10^{\wedge} 7$ & 287 & 8,598 \\
\hline HTR1B & 3,484 & 9,048 & 14,452 & $1,32^{*} 10^{\wedge} 4$ & $5,34^{*} 10^{\wedge} 6$ & $1,26^{*} 10^{\wedge} 6$ & 397563 & 8,994 \\
\hline BDKRB1 & 2,818 & 17,594 & 14,246 & $1,32 * 10^{\wedge} 4$ & $1,36^{*} 10^{\wedge} 6$ & $5,99 * 10^{\wedge} 7$ & 100127469 & 11,553 \\
\hline ITGB3 & 12,674 & 13,160 & 10,935 & $4,27^{*} 10^{\wedge} 6$ & $1,55^{*} 10^{\wedge} 6$ & $8,03 * 10^{\wedge} 7$ & 397063 & 12,256 \\
\hline FBN1 & 3,692 & 27,547 & 62,849 & $1,51 * 10^{\wedge} 4$ & $1,63^{*} 10^{\wedge} 6$ & $3,07^{*} 10^{\wedge} 7$ & 414836 & 31,363 \\
\hline THY1 & 38,753 & 32,743 & 31,302 & $3,01 * 10^{\wedge} 6$ & $1,39 * 10^{\wedge} 6$ & $5,59 * 10^{\wedge} 7$ & 100271931 & 34,266 \\
\hline
\end{tabular}

In this paper we focused on 54 genes that belong to "maintenance of location", maintenance of protein location in cell" and "maintenance of protein location" GO BP terms. These sets of genes were subjected to hierarchical clusterization procedure and presented as heatmaps (Fig. 1). The gene symbols, fold changes in expression, Entrez gene IDs and corrected p-values of these genes were shown in table $\mathbf{1}$.
The enrichment of each GO BP term was calculated as a z-score and shown on the circle diagram (Fig. 2).

Chosen GO BP terms contain 54 differently expressed genes. Therefore, we calculated the mean fold change ratio value of each gene between 7,15 and 30 days of culture. Based on that criteria, we chose the 10 most downregulated and 10 most upregulated genes for further analysis. 
D7/D1

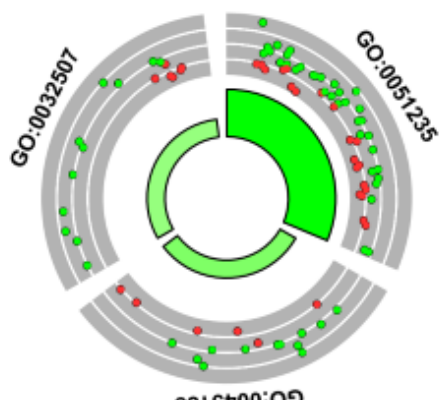

s8เ\$700:09

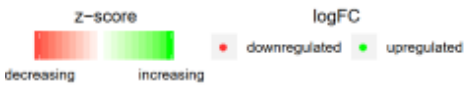

D15/D1

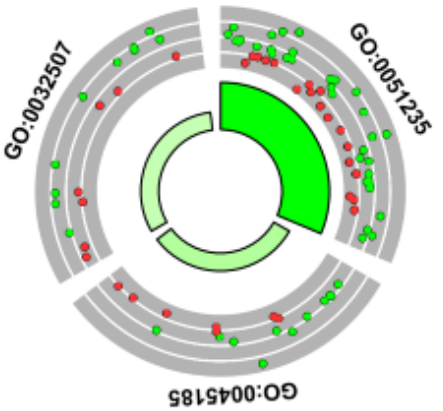

S8เS†00:09
D30/D1

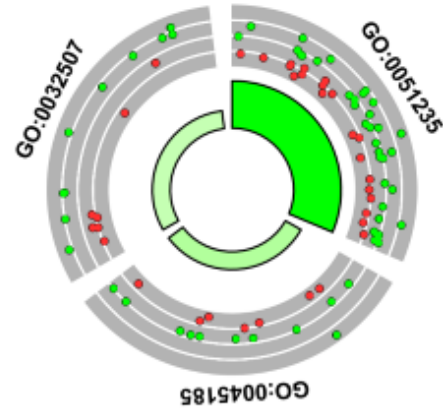

५8เৎ十00:09

\begin{tabular}{|c|c|}
\hline ID & Description \\
\hline GO:0051235 & maintenance of location \\
\hline GO:0045185 & maintenance of protein location \\
\hline GO:0032507 & maintenance of protein location in cell \\
\hline
\end{tabular}

FIGURE 2 The circle plot showing the differently expressed genes and z-score of the "maintenance of location", maintenance of protein location in cell" and " maintenance of protein location" GO BP terms. The outer circle shows a scatter plot for each term of the fold change of the assigned genes. Green circles display up- regulation and red ones down- regulation. The inner circle shows the z-score of each GO BP term. The width of the each bar corresponds to the number of genes within GO BP term and the color corresponds to the z-score

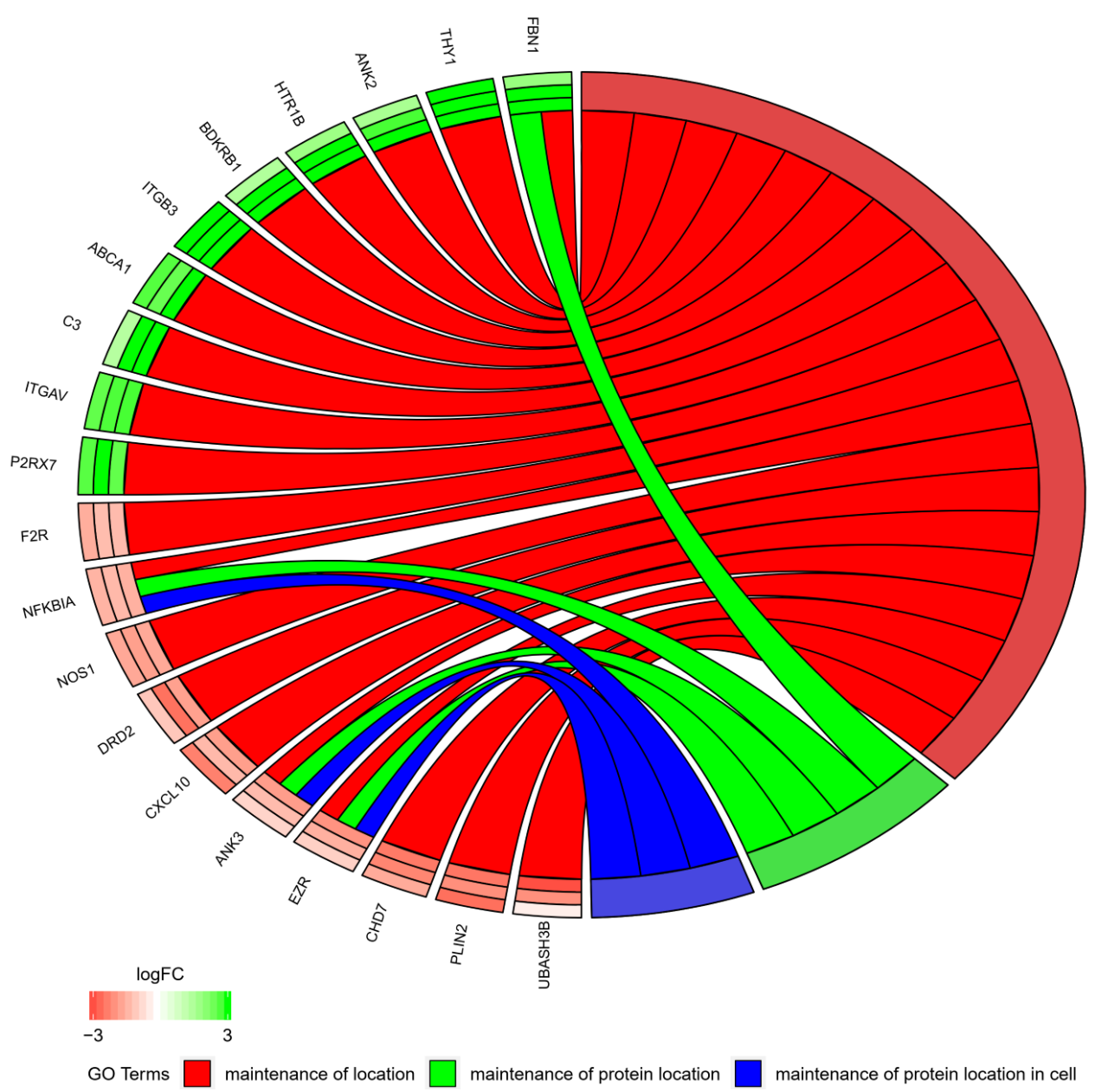

FIGURE 3 The representation of the mutual relationship of differently expressed genes that belongs to 20 chosen genes from "maintenance of location", maintenance of protein location in cell" and "maintenance of protein location" GO BP terms. The ribbons indicate which gene belongs to which categories. The middle circle represents logarithm from fold change (LogFC) between D7/D1, D15/D1 and D30/D1 respectively. The color of each block corresponds to the LogFC of each gene (green - upregulated, red - downregulated). The genes were sorted by logFC from most to least changed gene 


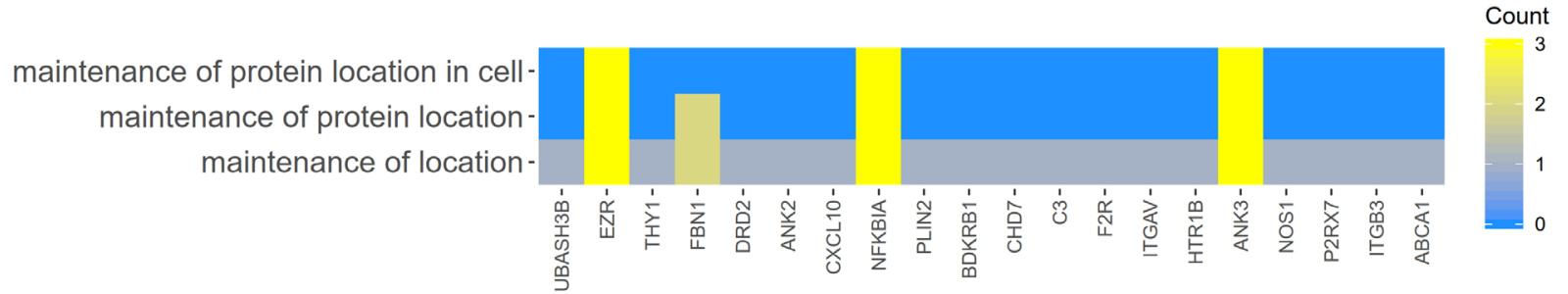

FIGURE 4 Heatmap showing the gene occurrence between chosen 20 differently expressed genes that belongs to "maintenance of location", maintenance of protein location in cell" and " maintenance of protein location" GO BP terms. The yellow color is associated with gene occurrence in the GO Term. The intensity of the color is corresponding to amount of GO BP terms that each gene belongs to

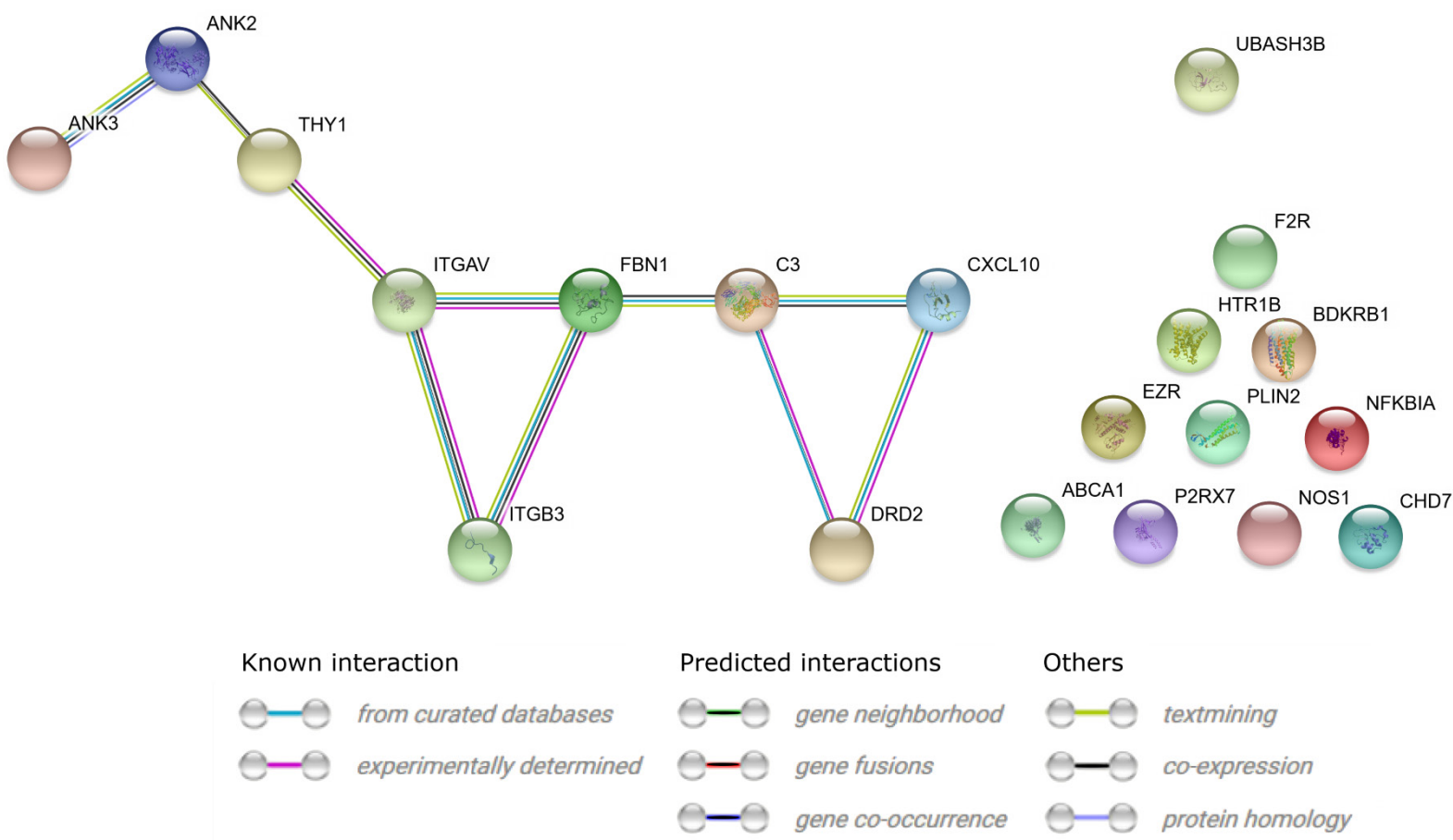

FIGURE 5 STRING-generated interaction occurrence between 20 chosen differently expressed genes that belongs to the "maintenance of location", maintenance of protein location in cell" and " maintenance of protein location" GO BP terms. The intensity of the edges reflects the strength of interaction score

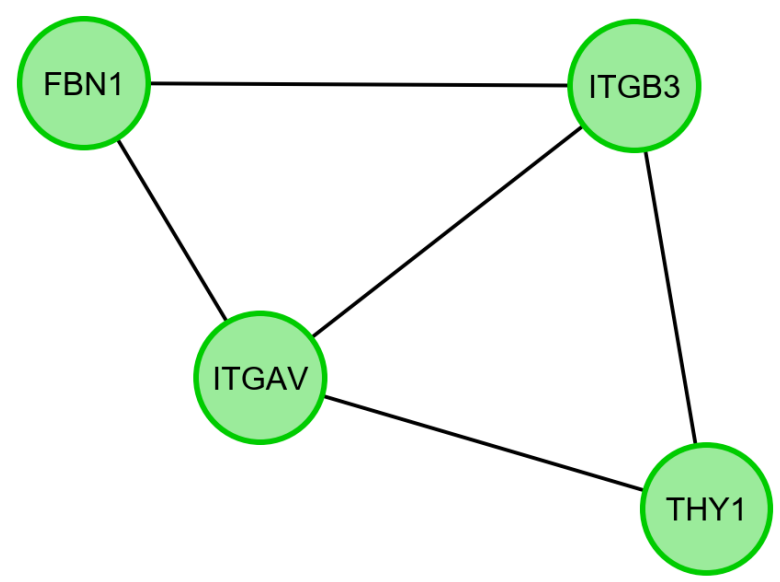

FIGURE 6 Functional interaction (FI) between 20 chosen differently expressed genes that belongs to the "maintenance of location", maintenance of protein location in cell" and " maintenance of protein location" GO BP terms. In following figure "->" stands for activating/catalyzing, "-|" for inhibition, "-" for FIs extracted from complexes or inputs, and "---" for predicted FIs 
In Gene Ontology database, genes that form one particular GO can also belong to other GO term categories. For this reason, we explored the gene intersections between the selected GO BP terms. The relation between those GO BP terms was presented as circle plot (Fig. 3) as well as heatmap (Fig. 4).

STRING interaction network was generated among the differentially expressed genes belonging to each of the selected GO BP terms. Using such prediction method provided us with a molecular interaction network formed between protein products of the studied genes (Fig. 5). Finally, we investigated the functional interactions between the chosen genes with REACTOME FIViz app to the Cytoscape 3.6.0 software. The results were shown in (Fig. 6).

\section{Discussion}

Whole transcriptome profiling analysis with the use of Affymetrix microarray revealed significant changes in gene expression of porcine oviductal epithelial cells during long term primary in vitro culture. The transcript levels were compared between day 1, 7, 15 and 30 of culture. We have found 54 differentially expressed genes belonging to three ontology groups: „maintenance of location”, „maintenance of protein location” and "maintenance of protein location in cell". However, most of them exert widespread functions throughout the body. In this paper, we focused on the 10 most upregulated genes and 10 most downregulated ones.

The group of the most downregulated genes after long term cultureincluded: PLIN2, UBASH3B, CHD7, DRD2, CXCL10, NOS1, EZR, NFKBIA, ANK3 andF2R, of which PLIN2 exhibited the most significant decrease in expression.PLIN2 (Perilipin 2) was found to be expressed in many cell types involved in lipid deposition and lipid turnover and is thought to be the marker of lipid droplet-containing cells [7]. PLIN2 expression occurs in porcine oocytes [8], with the study performed by Sastre et al. revealing its presence in oocytes and embryos of cattle as well. Moreover, it has been suggested that this protein promotes lipid accumulation [9].Therefore, this gene's downregulation in OECs may be an indication of lipid degradation after long term cultivation.

Another downregulated gene was ubiquitin-associated and SH3 domain-containing protein B (UBASH3B). This protein plays an important role during mitosis through interaction with ubiquitylated Aurora B, which drives it to microtubules [10].Thus, its function is not limited to oviductal cells. Similar situation occurs in regards toCHD7 gene, encoding chromodomain helicase DNA-binding protein 7, expression of which decreased as well. It has a wide range of functions in the entire organism, since its mutation results in CHARGE syndrome, characterized by abnormalities in many organs' development. CHD7 also acts as a transcriptional regulator, with studies conducted by Zentner et al. indicating that it is involved in rRNA synthesis [11]. Apart from that, they observed that depletion of this protein results in decreased cell proliferation and protein synthesis, which may also be the case during OECs long term in vitro culture.

DRD2 gene encodes two distinct isoforms of dopamine receptor D2, both of them engaged in signalling through dopamine in the nervous system [12]. Moreover, the study by Xu et al. revealed its expression in several other pig tissues, such as testes, back muscle and the oviduct, which is consistent with our results [13]. CXCL10 (C-X-C motif chemokine 10) encodes a chemokine, which is known to interact with DRD2 (Fig. 5).We have observed a significant decrease in its expression in our study. Apart from playing roles in development, homeostasis and function of the immune system [14], it is possible that CXCL10 is involved in early embryonic development, since the study by Tribulo et al. has shown that it is expressed in the bovine oviduct and endometrium [15].

Another downregulated gene, NOS1, encodes nitric oxide synthase 1 , which produces nitric oxide (NO). NO acts as a signalling molecule, exerting many functions throughout the body, one of them being the participation in the fertilization process. Kuo et al. demonstrated that NOS1 is expressed in both female and male gametes and that the NO signalling is necessary for an ovum activation [16].

The product of the next gene, that was significantly downregulated in our study, is engaged in many cellular proccesses. Ezrin, or cytovillin, encoded by EZR gene, is involved in cell adhesion, migration and the organization of cell surface structures, with its expression occuring in many epithelial cell types. It binds the cell membrane to actin filaments and is known to be present in microvilli [17]. Thus,EZR downregulation in OECs may be a reflection of changing cell morphology during long term culture. Ankyrin 3 (ANK3) is also a peripheral membrane proteinwith decreased expression in our study. However, it is located mainly at the axons' initial segment and the nodes of Ranvier and has been linked to wide spectrum of mental disorders [18].

$F 2 R$ encodes thrombin receptor, which is engaged in the regulation of thrombotic response. Thrombin cleaves fibrinogen to fibrin, also exerting cell-activating properties, such as platelet aggregation, which is crucial in the case of vascular injury [19]. This gene's expression hasn't been described in porcine OECs yet, with our study showing its downregulation after long term culture. NFKBIA, on the other hand, which also exhibited a decrease in expression in this study, has been linked with reproductive events, since Paciolla et al. suggested its involvement in bovine oocyte-to-embryo transition [20]. However, the protein encoded by this gene is typically engaged in immune responses and acts as an inhibitor of NF-kappa-B. 
In the course of this study we have also observed significant increase in several genes' expression, with this group including: ITGAV, C3, P2RX7, ABCA1, ANK2, HTR1B, BDKRB1, ITGB3, FBN1 and THY1.

Both ITGAV and ITGB3 belong to the family of integrins and interact with each other (Fig. 5), with integrin-based signalling having an influence on many genes' expression. These proteins ensure cell and extracellular matrix contact and are involved in many cellular events, such as migration, proliferation, survival and differentiation. Since $\alpha_{v} \beta_{3}$ integrin is located mainly in focal complexes, the upregulation of both of these subunits indicates that, after long term culture, the adherence of oviductal epithelial cells to each other increases [21]. Another upregulated gene, FBN1, encodes fibrillin 1, which is a part of extracellular microfibrils and is present mainly in connective tissue. Its interaction with ITGAV is well described, with the study conducted by Boregowda et al. revealing that integrin $\alpha_{\mathrm{v}}$ downregulation results in loss of FBN1 gene expression [22].

Fibrillin 1 also interacts with complement component 3 , which also exhibited a significant increase in expression during the course of this study. The complement system is engaged in innate and acquired immune responses and C3 is particularly involved in the lysis of microorganisms, opsonins production, mast cells and basophils degranulation and apoptotic cells clearance [23]. Additionally, C3 is known to be expressed in the human oviductal epithelial cells and can be converted into the iC3b, which is an embryotropic derivative. The study on mice and human oviductal epithelial cells performed by Lee et al. also revealed that E2 and preimplantation embryos enhance C3 expression, suggesting that this protein is involved in preimplantation embryo development as well [24].

THY-1 also exhibited an increase in expression and it is an another protein known to interact with ITGAV, probably participating in signal transduction after cell-cell interaction [25]. However, its primary function is the induction of T cells and NKT cell activation during the immune response. Apart from that, THY-1 is involved in many fundamental processes in the organism, since it influences cell migration, cell adhesion and tumour suppression. Therefore, its role is not limited to reproductive events [25]. THY-1 is thought to interact with another protein that was upregulated during this study, namely ankyrin 2 (Fig. 5). Similarly to THY1, ANK2 is not typically expressed in oviductal epithelial cells, since it is primarily localized in cardiomyocytes, with these gene's loss of function resultingin arrythmia [26].

Another upregulated gene was $P 2 R X 7$, which encodes an ionotropic cell surface ATP receptor and is involved in many biological processes. A study conducted by Rassendren et al. revealed its expression in many tissues, such as pancreas, liver, heart, thymus, brain, placenta, lung, testis, leukocytes and prostate [27]. Its role in fertilization has also been demonstrated in a study conducted on rat spermatozoa, which indicated that $\mathrm{P} 2 \mathrm{X} 7$ receptor is involved in acrosome reaction occurrencevia ATP binding [28].

We have also observed an increase in another ATP-binding protein level after long term OECs culture. ABCA1 belongs to the family of ATP binding cassette transporters that hydrolyse ATP to transport substrates across the cell membranes. Its main function is the participation in cellular lipid removal pathway [29] and its role has not yet been described in porcine OECs.

The last two upregulated genes, $H T R 1 B$ and $B D$ $K R B 1$ both encode receptors. HTR1B is a receptor for serotonin, therefore it is another protein that is not typical for oviductal epithelial cells. It acts as an autoreceptor on serotonin-containing neurons and heteroreceptor on neurons that lack serotonin, being implicated a role in pathophysiology of obsessive eating disorder, drug addiction, depression and anxiety has been [30]. BDKRB1, on the other hand, is a receptor for bradykinin, with the study conducted by Chai et al. demonstrating its expression in many tissues, e.g. uterus and ovaries. However it is believed that its expression mostly occurs under pathological conditions [31].

Summing up, our current results revealed a set of genes belonging to three ontology groups involved in maintenance of protein location in cells. However, most of these genes exert widespread functions in the organism and their role is not limited to reproductive events. It is also important to note that, during long term in vitro culture, the morphology and biochemistry of epithelial cells may change. Therefore, we conclude that differential expression of aforementioned genes may be a reflection of these changes.

\section{Acknowledgements}

This publication and its results are an outcome of a cooperation between Poznan University of Medical Sciences (Poznań, Poland) and Polish Ministry of Science and Higher Education, with Institute of Advanced Sciences Sp. z o.o. (Poznań, Poland), as a part of the "Professional PhD" programme.

\section{Corresponding author}

Michal Jeseta; Department of Obstetrics and Gynaecology, University Hospital and Masaryk University, Brno, Czech Republic; email: jeseta@gmail.com.

\section{Conflict of interest statement}

The authors declare they have no conflicts of interest.

\section{References}

1. Li S, Winuthayanon W. Oviduct: roles in fertilization and early embryo development. J Endocrinol. 2017;232:R1-26; DOI:10.1530/ JOE-16-0302.

2. Leese HJ. The formation and function of oviduct fluid. J Reprod Fertil. 1988;82:843-56.

3. White KL, Hehnke K, Rickords LF, Southern LL, Thompson DL, Wood TC. Early Embryonic Development in Vitro by Coculture with Oviductal Epithelial Cells in Pigs1. Biol Reprod. 1989;41:425-30; D0I:10.1095/ biolreprod41.3.425. 
4. Huang DW, Sherman BT, Tan Q Collins JR, Alvord WG, Roayaei J, Stephens R, Baseler MW, Lane HC, Lempicki RA. The DAVID Gene Func tional Classification Tool: a novel biological module-centric algorithm to functionally analyze large gene lists. Genome Biol. 2007;8:R183; DOI:10.1186/gb-2007-8-9-r183.

5. Walter W, Sánchez-Cabo F, Ricote M. GOplot: An R package for visually combining expression data with functional analysis. Bioinformatics. 2015;31:2912-4; DOI:10.1093/bioinformatics/btv300.

6. von Mering C, Jensen LJ, Snel B, Hooper SD, Krupp M, Foglierini M, Jouffre N, Huynen MA, Bork P. STRING: known and predicted protein-protein associations, integrated and transferred across organisms. Nucleic Acids Res. 2004;33:D433-7; DOI:10.1093/nar/gki005.

7. Heid HW, Moll R, Schwetlick I, Rackwitz HR, Keenan TW. Adipophilin is a specific marker of lipid accumulation in diverse cell types and diseases. Cell Tissue Res. 1998;294:309-21.

8. Zhang R, Fu X, Jia B, Liu C, Cheng K, Zhu S. Expression of Perilipin 2 (PLIN2) in Porcine Oocytes During Maturation. Reprod Domest Anim. 2014;49:875-80; DOI:10.1111/rda.12386

9. Sastre D, Costa NN da, Sá ALA de, Conceição SDB, Chiaratti MR, Adona PR, Guemra S, Meirelles FV, Santos S do SD, Sena L, Ohashi OM, Santos EJM dos, Miranda M dos S. Expression of PLIN2 and PLIN3 during oocyte maturation and early embryo development in cattle. Theriogenology. 2014;81:326-31; DOI:10.1016/J.THERIOGENOLOGY.2013.10.002.

10. Krupina K, Kleiss C, Metzger T, Fournane S, Schmucker S, Hofmann K, Fischer B, Paul N, Porter IM, Raffelsberger W, Poch O, Swedlow JR, Brino L, Sumara I. Ubiquitin Receptor Protein UBASH3B Drives Aurora B Recruitment to Mitotic Microtubules. Dev Cell. 2016;36:63-78; DOI:10.1016/j.devcel.2015.12.017.

11. Zentner GE, Hurd EA, Schnetz MP, Handoko L, Wang C, Wang Z, Wei C, Tesar PJ, Hatzoglou M, Martin DM, Scacheri PC. CHD7 functions in the nucleolus as a positive regulator of ribosomal RNA biogenesis. Hum Mol Genet. 2010;19:3491-501; DOI:10.1093/hmg/ddq265.

12. Usiello A, Baik J-H, Rougé-Pont F, Picetti R, Dierich A, LeMeur M, Piazza $\mathrm{PV}$, Borrelli E. Distinct functions of the two isoforms of dopamine D2 receptors. Nature. 2000;408:199-203; DOI:10.1038/35041572.

13. Xu HP, He XM, Fang MX, Hu YS, Jia XZ, Nie QH, Zhang XQ. Molecular cloning, expression and variation analyses of the dopamine D2 re ceptor gene in pig breeds in China. Genet Mol Res. 2011;10:3371-84 DOI:10.4238/2011.December.5.6

14. Zlotnik A, Yoshie 0. Chemokines: a new classification system and their role in immunity. Immunity. 2000;12:121-7; DOI:10.1016/ S1074-7613(00)80165-X.

15. Tríbulo P, Siqueira LGB, Oliveira LJ, Scheffler T, Hansen PJ. Identification of potential embryokines in the bovine reproductive tract. J Dairy Sci. 2018;101:690-704; DOI:10.3168/jds.2017-13221.

16. Kuo RC, Baxter GT, Thompson SH, Stricker SA, Patton C, Bonaventura J, Epel D. NO is necessary and sufficient for egg activation at fertilization. Nature. 2000;406:633-6; DOI:10.1038/35020577.

17. Majander-Nordenswan P, Sainio M, Turunen O, Jääskeläinen J, Carpén O, Kere J, Vaheri A. Genomic structure of the human ezrin gene. Hum Genet. 1998; DOI:10.1007/s004390050888.

18. Iqbal Z, Vandeweyer G, van der Voet M, Waryah AM, Zahoor MY, Besseling JA, Roca LT, Vulto-van Silfhout AT, Nijhof B, Kramer JM, Van der Aa N, Ansar M, Peeters H, Helsmoortel C, Gilissen C, Vissers LELM, Veltman JA de Brouwer APM, Frank Kooy R, Riazuddin S, Schenck A, van Bokhoven $\mathrm{H}$, Rooms L. Homozygous and heterozygous disruptions of ANK3: at the crossroads of neurodevelopmental and psychiatric disorders. Hum Mo Genet. 2013;22:1960-70; DOI:10.1093/hmg/ddt043.

19. Coughlin SR, Vu TK, Hung DT, Wheaton VI. Characterization of a functional thrombin receptor. Issues and opportunities. J Clin Invest. 1992;89:351-5; DOI:10.1172/JCI115592.

20. Paciolla M, Boni R, Fusco F, Pescatore A, Poeta L, Ursini M V., Lioi MB, Miano MG. Nuclear factor-kappa-B-inhibitor alpha (NFKBIA) is a developmental marker of NF- B/p65 activation during in vitro oocyte maturation and early embryogenesis. Hum Reprod. 2011;26:1191-201 DOI:10.1093/humrep/der040.

21. Morgan MR, Byron A, Humphries MJ, Bass MD. Giving off mixed signals --distinct functions of alpha5beta1 and alphavbeta3 integrins in regulating cell behaviour. IUBMB Life. 2009;61:731-8; DOI:10.1002/iub.200.

22. Boregowda RK, Krovic BM, Ritty TM. Selective integrin subunit reduction disrupts fibronectin extracellular matrix deposition and fibrillin 1 gene expression. Mol Cell Biochem. 2012;369:205-16; DOI:10.1007/ s11010-012-1383-y.

23. Reis ES, Falcao DA, Isaac L. Clinical Aspects and Molecular Basis of Primary Deficiencies of Complement Component C3 and its Regulatory Proteins Factor I and Factor H. Scand J Immunol. 2006;63:155-68; DOI:10.1111/j.1365-3083.2006.01729.x.
24. Lee Y-L, Cheong AWY, Chow W-N, Lee K-F, Yeung WSB. Regulation of complement-3 protein expression in human and mouse oviducts. Mol Reprod Dev. 2009;76:301-8; DOI:10.1002/mrd.20955.

25. Park K, Kim J, Choi C-Y, Bae J, Kim S-H, Kim Y-H, Chun T. Molecular Cloning and Expression Analysis of Pig Cd90. Anim Biotechnol. 2016;27:133-9 DOI:10.1080/10495398.2015.1129630.

26. Koenig SN, Mohler PJ. The evolving role of ankyrin-B in cardiovascular disease. Hear Rhythm. 2017;14:1884-9; DOI:10.1016/j. hrthm.2017.07.032.

27. Rassendren F, Buell GN, Virginio C, Collo G, North RA, Surprenant A. The Permeabilizing ATP Receptor, P2X . J Biol Chem. 1997;272:5482-6; DOI:10.1074/jbc.272.9.5482

28. Torres-Fuentes JL, Rios M, Moreno RD. Involvement of a P2X7 Receptor in the Acrosome Reaction Induced by ATP in Rat Spermatozoa. J Cell Physiol. 2015;230:3068-75; DOI:10.1002/jcp.25044.

29. Lawn RM, Wade DP, Garvin MR, Wang X, Schwartz K, Porter JG, Seilhamer JJ, Vaughan AM, Oram JF. The Tangier disease gene product ABC1 controls the cellular apolipoprotein-mediated lipid removal pathway. Clin Invest. 1999;104:R25-31; DOI:10.1172/JCI8119.

30. Svenningsson P, Chergui K, Rachleff I, Flajolet M, Zhang X, El Yacoubi M Vaugeois J-M, Nomikos GG, Greengard P. Alterations in 5-HT1B Receptor Function by p11 in Depression-Like States. Science (80- ). 2006;311:7780; DOI:10.1126/science.1117571.

31. Chai KX, Ni A, Wang D, Ward DC, Chao J, Chao L. Genomic DNA Sequence, Expression, and Chromosomal Localization of the Human B1 Bradykinin Receptor Gene BDKRB1. Genomics. 1996;31:51-7; DOI:10.1006/ geno.1996.0008. 